\title{
Study on Performance of Silicon Ignitiors based on Energetic Chip of Porous Silicon
}

\author{
Lan Liu ${ }^{a}$, Yan Xue ${ }^{\mathrm{b}}$, Ruizhen Xie ${ }^{\mathrm{c}}$, Xiaoming Ren ${ }^{\mathrm{d}}$ and Kewei Li \\ National Key Laboratory of Applied Physics and Chemistry, Shaanxi Applied Physics-Chemistry Research Institute, Xi'an, \\ China,710061 \\ a383131204@qq.com, bxueyan213@163.com, ${ }^{\text {cxieruizhen@126.com, }{ }^{d} \text { rxm_2003@163.com@163.com, }{ }^{9} 994060346 @ q q . c o m}$
}

\begin{abstract}
In order to study the igniton properties of the silicon ignitior, firing sensitivity test with output charge B-KNO3 were carried out, and the igniton properties was studied as well. The result indicates that the energetic chip can ignite B-KNO3 which burns sufficiently. The $50 \%$ firing voltage was $21.3 \mathrm{~V}$, standard deviation was 1.87 , the $99.9 \%$ point was $27.1 \mathrm{~V}$.
\end{abstract}

Keywords: porous silicon, energetic chip, silicon ignitior, electrochemical etching.

\section{Introduction}

As the modern weaponry developed toward miniaturization, intelligent, integrated, traditional Pyrotechnics can not meet the requirements[1,2]. Silicon ignitiors are based on the concept of MEMS manufacturing. The porous silicon chips have micronano-scale precision and the microscale planar structure which greatly reduced the volume and the weight of initiator[3,4]. The performance of porous silicon energetic chip can be adjust by different concentration of $\mathrm{NaClO} 4 /$ methanol solution[5,6].

Aiming at the need of integration development of pyrotechnics, the design and manufacture of silicon ignitior were researched, which include the whole design, the manufacture of porous silicon sequence and its performance. The design principle of initiators based on MEMS will support the development of mocro arms initiation, ignition and work.

\section{Experiment}

\subsection{Reagent and apparatus}

HF acid,analytical, XI'an chemical Reagent factory; Ethanol, Sichuan xilong Chemical Co.;Bno3,Xi'an213; P-type silicon, crystal-100,Resistivity of $0.02 \sim 0.03 \Omega \cdot \mathrm{cm}$, thickness: $300 \pm 15 \mu \mathrm{m}$, Shanghai Junhe electronic materials Co.; platinum electrodes, pureity $99.95 \%$, diameter 70mm, Shanghai dingfu metal materials Co.; NaClO4, analytical, Tianjin fucheng chemical Reagent factory; current loop, and width $: 35 \mathrm{MHz}$, measuring range: :0 500A, rise time :10ms.

\subsection{Experiment equipment}

The schematic diagram of ignition test system shown in Figure 1, which is made up of oscilloscope, optical probes, current loop and switch. The fire sensitivity tests of porous silicon ignitors were carried out and the range of voltage is from $10 \mathrm{~V}$ to $50 \mathrm{~V}$. 


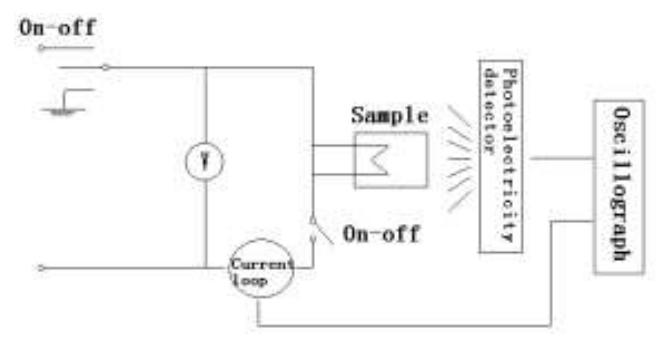

Fig.1. The test system of porous silicon energetic chip

\subsection{Preparation of test sample}

\subsubsection{Design of porous silicon ignitor}

Porous silicon ignitor was made of shell, output charge, energetic porous silicon chip, the energetic chip and electrode plug. The bridge film was produced through the process of sputtering, photolithography and wet etching. The micro energetic chip was made by in-situ charge method. The chip was stick to the groove in electrode plug. The sctech map showed in Figure 2.

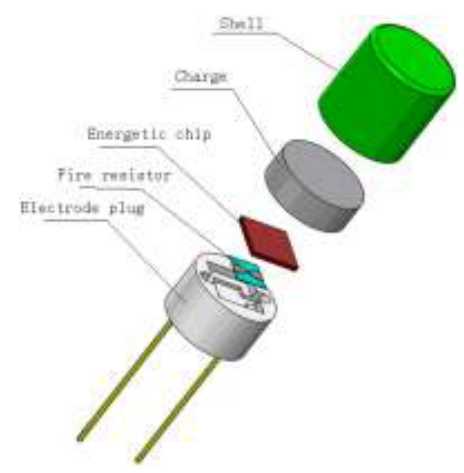

(a)Structure of silicon ignitior

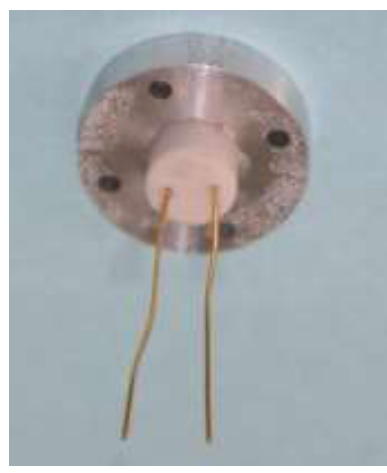

(b)Sample of silicon ignitior

Fig.2. Structure and sample of silicon ignitior and Silicon ignitior

\subsubsection{Design of charge}

The charge was $74 \mathrm{mg} \mathrm{B} / \mathrm{KNO} 3$ pellet, whose charge size was $\Phi 4.2 \mathrm{~mm} \times 3.8 \mathrm{~mm}$ and density was $1.4 \mathrm{~g} / \mathrm{cm} 3$.

2.3.3 Preparation of porous silicon energetic chip

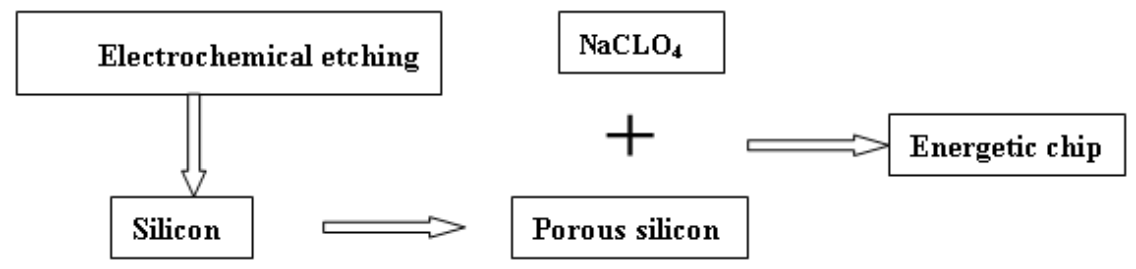

Fig.3 Preparation process of energetic chip

The principle of porous silicon energetic chip was showed in Figure 3. The silicon was prepared by electronchemical etching to form a sponge-like structure. The saturated solution of $\mathrm{NaClO} 4$ was added to the porous silicon to make the porous silicon energetic chip. The porous silicon was produced by electrochemical etching of silicon in $\mathrm{HF} / \mathrm{ethanol}(\mathrm{HF} / \mathrm{CH} 3 \mathrm{CH} 2 \mathrm{OH}=1: 1)$ electrolyte by five step constant current etching method. 
Table 1. The parameter of Porous silicon Preparation

\begin{tabular}{|c|c|c|c|c|c|}
\hline & Step 1 & Step 2 & Step 3 & Step 4 & Step 5 \\
\hline Current(A) & 2.8 & 2.4 & 2.0 & 1.6 & 1.3 \\
\hline Etching time(min) & 20 & 10 & 30 & 10 & 50 \\
\hline
\end{tabular}

Placed porous silicon on the hot plate. Added saturated $\mathrm{NaCLO} 3$ to the porous silicon to made the porous silicon energetic chip. The temperature of hot plate controlled at $70^{\circ} \mathrm{C} \sim 90^{\circ} \mathrm{C}$ and blowing hot air to the porous silicon 5 8second.Repeated dropping 3 5times. Carbinol volatilized, and oxidant aggregated in the porous silicon. The structural of porous silicon energetic chip shown in Figure 4.

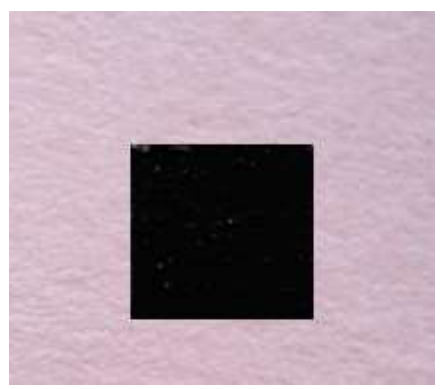

Fig.4. Image of porous silicon energetic chip

\section{Results and discuss}

The firing sensitivity was tested according to LangLie Method[7,8], firing current was less than 1.7A, firing voltage was less than $15 \mathrm{~V}$, ignition time was millisecond level, result can be seen from the table 2 :

Table 2. Firing voltage of porous silicon energetic chip

\begin{tabular}{|c|c|c|c|c|}
\hline number & sample siza & $\begin{array}{c}50 \% \text { firing } \\
\text { voltage }\end{array}$ & $\begin{array}{c}99.9 \% \text { response } \\
\text { point }\end{array}$ & $\begin{array}{c}\text { standard } \\
\text { deviation }\end{array}$ \\
\hline 1 & 12 & $21.3 \mathrm{~V}$ & $27.1 \mathrm{~V}$ & 1.87 \\
\hline 2 & 15 & $14.4 \mathrm{~V}$ & $20.8 \mathrm{~V}$ & 2.09 \\
\hline 3 & 17 & $18.6 \mathrm{~V}$ & $17.9 \mathrm{~V}$ & 1.23 \\
\hline
\end{tabular}

The firing sensitivity was tested according to LangLie Method, $50 \%$ firing voltage was less than $15 \mathrm{~V}$, test results wa shown in Figure 5. The image of porous silicon energetic chip after firing was shown in figure 6, BKNO3 effected completely shown in figure 6 .

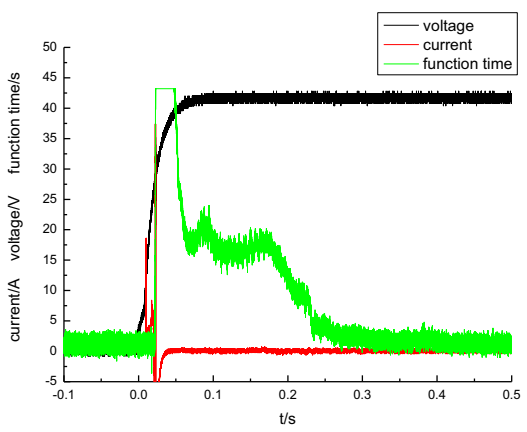

Fig.5. Curve of firing voltage、 current and function time change 


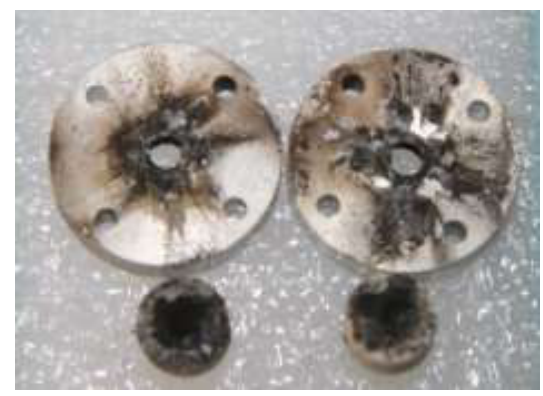

Fig.6. image of porous silicon energetic chip after firing of porous silicon energetic chip

\section{Conclusion}

Paper used electronchemical etching method to etched the silicon into a sponge-like structure.Added saturated solution of $\mathrm{NaCLO} 3$ to the porous silicon to made the porous silicon energetic chip. LangLie Method used to test the firing sensitivity of porous silicon energetic chip ignition, results indicated:

(1)Combined with the technics of MEMS, used electronchemical etching method to etched porous silicon energetic chip.Company with MEMS Ni/Cr-bridges and charge to facture the silicon ignitiors.

(2)The energetic chip can ignited B-KNO3 which burns sufficiently, text indicated porous silicon energetic chip can ignited charge and burning sufficiently.

(3)LangLie Method used to test the firing sensitivity of porous silicon energetic chip ignition, the $50 \%$ firing voltage $21.3 \mathrm{~V}$, standard deviation 1.87 the $99.9 \%$ point $27.1 \mathrm{~V}$.

\section{References}

1. Y. Xue, B. Lu, Properties of Energetic Materials Based on Nano-Porous Silicon. Chinese Journal of Energetic Materials, 18,5(2010)

2. Churaman W., L. Currano, C. Becker, Initiation and reaction tuning of nanoporous energetic silicon. Journal of Physics and Chemistry of Solids, 71, 2(2010)

3. Joachim Diener, Egon Gross, Dimitri Kovalev etal., Nanostructured Reactive Substance and Process for Producing the Same, US patent 0148569, (2003)

4. D. Clement etc, Highly explosive nanosilicon-based composite materials.phys.atat.sol, (a) 202, No.8 2005.

5. L. Liu, Y. Xue, etal., Influence of Electrochemical Etching Parameters on morphology of porous silicon, Mechanical Science and Engineering, 12(2014)

6. S.X. Wang, R. Q. Shen, Y.H. Ye, Preparation and ProPerties of Porous Silicon Energe Chips, Chinese Journal of Energetic Materials, 18, 5(2010)

7. R. Zhang, D.X. FU, etc. A Calculation Instrument for the Sensitivity Test of Initiating Explosive Device. Initiators\&Pyrotechnics, 1, 2(2009)

8. D.X. FU, R. Zhang. etc. Estimation and Its Applicability of D-Optimality-Based Sensitivity Test under Different Prior Information. Chinese Journal of Energetic Materials, 17, 3(2009) 